\title{
Are rare earths part of a bright future for lighting and displays?
}

\author{
By Eva Karatairi \\ Feature Editor Henning Höppe
}

A re we running out of europium? This is the kind of question that generates blank looks. The slightest hint, however, that smartphones cannot be upgraded would generate an uproar. Europium belongs to the lanthanides group, which are part of the 17 rare-earth elements. Many of them face high risk for shortage in the near future, something that raises concerns over the future of high-tech applications.

Screens in smartphones and laptops need rare earths for the expression of red, blue, and green colors. Rare earths are also found in phone circuits. Neodymium is necessary for the permanent magnets in speakers - the same category of powerful magnets found in electric motors and wind turbines. If rare earths are in short supply, is there a possibility that we keep in our pockets the latest version of a mobile phone?

A look at the abundance of rare earths in the Earth's crust could reassure one's fears, as it would reveal that they are not actually so rare, at least in terms of occurrence in the outermost solid layer of our planet. Even thulium, the least abundant among them, is more plentiful than gold and silver. It is rare, however, to find them in exploitable concentrations.

Not only are the identified resources restricted, but onethird of the global reserves are in China, a country that also controls around $90 \%$ of the world's rare-earth oxides production. Furthermore, demand is expected to increase steadily in the years to come, partly because of the foreseen advances in green technologies. These facts combined together reveal the reasons behind the serious concerns over their availability.

High purity of rare-earth metals is important for many advanced applications, but separating rare earths from the oxides they form - in the minerals they are found in-is a costly procedure and not an easy one. The reason that makes their extraction, purification, and recycling processes so difficult is the same one that gives rise to their unique spectroscopic and magnetic properties. All 15 lanthanide elements have the same electron arrangement in their outer shell $\left(6 s^{2}, 5 d^{1}\right)$ and thus a very similar chemical character.

Apart from these outer $6 s$ and $5 d$ shells, two more filled orbitals ( $5 p$ and $5 s)$ shield the $4 f$ subshell of their atoms, except for $\mathrm{La}^{3+}$ and $\mathrm{Lu}^{3+}$. The way electrons occupy this $4 f$ orbital and the number of unpaired electrons in it differentiates one element from the other. Furthermore, transitions of electrons between the $f$ orbitals and $d$ and $f$ orbitals ( $f-f$ and $d-f$ transitions, respectively) result in the luminescent character of rare earths.

With major applications in the display and lighting industry, phosphors have proven to be a perfect field for rare earths to unfold their extraordinary optical properties: they are among the few materials whose emission spectra are very characteristic and hardly influenced by their chemical environment.

"The field of rare-earth-based luminescent materials is burgeoning because all trivalent lanthanide ions, barring lanthanum and lutetium, behave as ideal wavelength converting devices," said Jean-Claude Bünzli, a professor at École Polytechnique Fédérale de Lausanne and an expert in luminescent lanthanide materials. "They exhibit narrow emission bands that cover the entire spectral range from UV to visible and near-infrared (up to $3 \mu \mathrm{m}$ ), while trivalent cerium and some divalent ions such as europium or samarium display broad but widely tuneable $d$-f emission," he added.

In phosphors based on trivalent rare-earth ions, luminescence is due to $4 f-4 f$ transitions (forbidden absorptions, ) which are almost independent from the host matrix. Divalent rare earths exhibit $4 f-5 d$ transitions, allowed by spin, and hence are intense. In this case, the participation of $5 d$ transitions in the luminescence process is strongly influenced by the crystal field and the symmetry of the surrounding lattice. However, it enables tuning of emission wavelength, making these materials promising for luminescent tubes or white light-emitting diodes (WLEDs).

Depending on how much time the emission of radiation lasts, phosphors are divided into phosphorescent and fluorescent materials. The first category shows a slower ( $>1 \mathrm{~ms})$ emission decay than the latter, in which case materials lose their brightness in a time scale of nanoseconds. The emission of light in phosphors is the final step in a mechanism that starts with the absorption of energy from an external source, enough to raise electrons to upper electronic levels. The relaxation of the electrons, back to their basic energetic states, is accompanied by the release of energy in the form of photons

The excitation source is another parameter that often defines the type of luminescence. For example, photoluminescence is the result of excitation through UV light (fluorescent lamps, energyefficient lighting systems); cathodoluminescence results from the excitation of electrons (TVs, monitors); and electroluminescence is connected with excitation from an electric field (LEDs, plasma 
displays, and LCDs). Most of these technologies include phosphors based on rare earths.

Andries Meijerink, professor of chemistry at the University of Utrecht and an expert in the field, expects a rapid transition toward LED lighting in the next five years. "The transition is driven by the discovery of new red phosphors that enable efficient warm white LEDs, replacing unattractive cool white LEDs," added Meijerink. "Most LED phosphors converting blue light into (warm) white light rely on rare-earth ions, specifically europium and cerium," said Meijerink.

An example of phosphors developed in Meijerink's laboratory can be seen in the photo. The bright yellow powder is YAG:Ce, the first phosphor applied by Nichia in 1996 to make WLEDs by combining YAG:Ce with a blue LED. It is still present in almost every WLED (e.g., in mobile phones, it is possible to see this yellowish powder when looking at the LED in the back). Green luminescent material is a $\mathrm{Eu}^{2+}$ phosphor. The red powder is one of the newest phosphors, a fluoride doped with $\mathrm{Mn}^{4+}$ giving narrow band red emission. This phosphor is combined with the green luminescent phosphor in warm WLEDs and WLED backlights for displays.

But this red luminescent material is a phosphor that does not contain any rare earths. "Efficient narrow band red emission from $\mathrm{Mn}^{4+}$ in fluorides and from quantum dots enables a wider color gamut, higher efficiency, and better color rendering, which is attractive for displays and lighting," said Meijerink. "Warm white LEDs and LCD displays made with these new phosphors have entered the market on a significant scale in 2016. These new color converters may continue to replace rare-earth phosphors."

Bünzli agreed: “Although phosphors for lighting perform best with rare earths, depending on availability and prices, they might be partially replaced with less compelling and more easily accessible materials."

Colin Humphreys, director of research in the Department of Materials Science and Metallurgy at the University of Cambridge, took the conversation one step further: "Hopefully, next-generation LEDs for lighting will not use phosphors at all. If we can increase the efficiency of green and yellow LEDs, then we can produce white light by mixing red, green, blue, and yellow LEDs without using any phosphors."
Oscar Klier, who is working on his $\mathrm{PhD}$ thesis under the supervision of Armin Reller in the Resource Strategy Department of the Universität Augsburg in Germany, is more optimistic. "At the moment, phosphor-converted LEDs provide the most efficient way to generate white light in solid-state lighting." The problem is that the use of phosphors is associated with a waste of energy "due to inherent conversion losses caused by Stokes shift," said Klier. "By omission of an energy-absorbing phosphor layer in the path of light emanating from the actual LED die, higher values in luminous efficacy can be reached."

Klier believes that phosphor-related difficulties such as thermal quenching or unwanted light scattering can also be avoided. "The reason why multi-color LED arrays combining red, green, and blue LEDs to generate white light have not replaced systems using phosphors is the socalled efficiency gap for LEDs, emitting in the spectral region ranging from yellow to green. With the introduction of novel semiconductor compounds and highly specialized methods of fabrication, this drawback in efficiency might be overcome soon," he said.

Furthermore, the rareearth fever around the globe is not directly related to the use of rare earths in luminescent materials. The market value for phosphors in 2015 was onethird of the rare-earth market, but in terms of volume, they are a rather modest application (about 7\%), according to the study "Rare Earths and the Balance Problem," published by Binnemans and Jones. When scientists were asked if they believe that the risk for shortage in rare-earth supply in the near future will hamper the advanced lighting and screen market, the picture that emerges is far from dark.

According to Meijerink, "The rapid growth of the white LED market will not be hampered by a shortage of rare earths. The amount of rare-earth phosphors in a white LED is only $1 \mathrm{mg}$, a thousand times less than the $1 \mathrm{~g}$ of rare earths in (compact) fluorescent tubes. Even when China will further limit the export of rare earths, the small amounts required for white light LEDs will be available from alternative sources. There is no risk of a rare-earth shortage for LED lighting."

"Some applications require a little amount of rare earths and are not really concerned by criticality (or cost) aspects," Bünzli said. "In spite of the advent of other performing luminescent materials and even though the availability of some rare-earth elements (yttrium, europium, terbium) is limited, their unique spectroscopic properties will continue to be exploited." 


\section{MRS ENERGY Q}

\section{SUSTAINABILITY}

\section{A Review Journal}

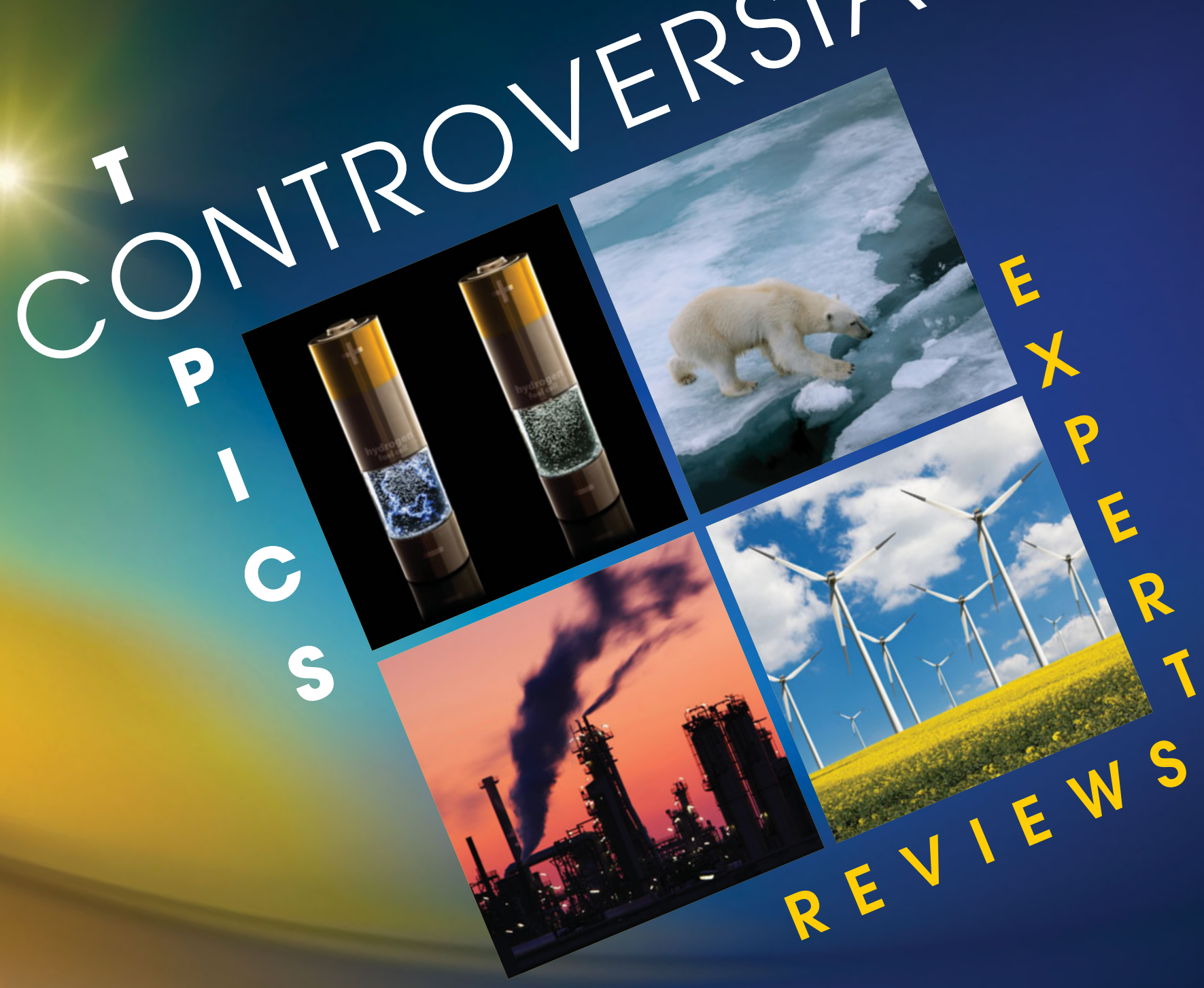

MRS Energy \& Sustainability - A Review Journal publishes reviews on key topics in materials research and development as they relate to energy and sustainability. Review topics include new R\&D of both established and new areas; systems integration; and objective application of economic, sociological and governmental models, enabling research and technological developments. The reviews are set in an integrated context of scientific, technological and sociological complexities relating to environment and sustainability.
㟧 David S. Ginley

National Renewable Energy Laboratory, USA

0

$\geq$ David Cahen

के Weizmann Institute of Science, Israel

을 Elizabeth A. Kócs

ㅍ. University of Illinois at Chicago, USA 